\title{
Backpropagation Neural Network Artificial Intelligence Algorithm-Based Magnetic Resonance Imaging Image Feature Analysis in the General Anesthesia Hip Arthroplasty
}

\author{
Yufang Li $\mathbb{D},{ }^{1}$ Xin Wang $\mathbb{C}^{2},{ }^{2}$ Qian Zhao $\mathbb{D}^{1},{ }^{1}$ Xiaoqing Zhang $\mathbb{D},{ }^{1}$ and Manyun Bai $\mathbb{D}^{1}$ \\ ${ }^{1}$ Department of Anesthesiology, The Fourth Hospital of Changsha (Changsha Hospital of Hunan Normal University), \\ Changsha 410006, Hunan, China \\ ${ }^{2}$ Department of Musculoskeletal Cancer, Hunan Cancer Hospital (The Affiliated Cancer Hospital of Xiangya School of Medicine, \\ Central South University), Changsha 410013, Hunan, China
}

Correspondence should be addressed to Manyun Bai; 1751030141@xzyz.edu.cn

Received 10 July 2021; Revised 12 August 2021; Accepted 18 August 2021; Published 25 August 2021

Academic Editor: Gustavo Ramirez

Copyright (c) 2021 Yufang Li et al. This is an open access article distributed under the Creative Commons Attribution License, which permits unrestricted use, distribution, and reproduction in any medium, provided the original work is properly cited.

\begin{abstract}
Objective. This study aimed to present an investigation of the clinical significance of magnetic resonance imaging (MRI) images obtained based on the backpropagation neural network (BPNN) artificial intelligence algorithm for hip arthroplasty under general anesthesia. Methods. In this study, a case-review method was used to collect 100 patients requiring total hip replacement. They were then randomly divided into an observation group and a control group. Based on the neural network algorithm, the images of the two groups of patients were analyzed to judge their accuracy. Then the sensitivity, specificity, and accuracy of MRI images based on neural algorithms were compared with those processed by radiologists. Results. It was found that MRI processed by BP neural network had good accuracy in the diagnosis of hip joint diseases compared with CT. Meanwhile, the images processed by BP neural network had good specificity and accuracy compared with the images processed by radiologists. Conclusion. Imaging images obtained by BPNN artificial intelligence algorithm were more accurate than CT images, which had more guiding value for surgeons in operation.
\end{abstract}

\section{Introduction}

Hip arthroplasty is one type of artificial joint replacement. The indications for hip arthroplasty are mainly osteoarthritis, aseptic necrosis of the bone, certain hip fractures, rheumatoid arthritis, traumatic arthritis, benign and malignant bone tumors, ankylosing spondylitis, etc. [1]. Clinical data have shown that the incidence of osteoarthritis in $45-65$-year-olds is up to $30 \%$ and in 65 -year-olds can reach $60 \%$ [2-4]. The main purpose of an artificial joint replacement is to relieve joint pain, correct deformity, and restore and improve joint motor function $[5,6]$. In summary, there are indications for joint arthroplasty whenever there are X-ray signs of joint destruction, accompanied by moderate to severe persistent joint pain and dysfunction that cannot be relieved by various other nonsurgical treatments [7]. General anesthesia has better anesthesia and analgesia effect on elderly patients undergoing hip arthroplasty and has little effect on postoperative cognitive function [8].

Magnetic resonance imaging (MRI) has been applied in the imaging diagnosis of various systems in the whole body, and the best results are the brain, spinal cord, cardiac vessels, joint bones, soft tissue, and pelvic cavity [9]. Compared with other imaging examinations, MRI has many advantages, such as no ionizing radiation damage to the human body, the ability to obtain primary three-dimensional cross-sectional imaging, and the clear display of soft tissue structure $[10,11]$. Clinical data have suggested that MRI can more specifically reflect the early necrosis of the femoral head, which has a great role in the preoperative diagnosis of hip arthroplasty [12]. With the development of artificial intelligence algorithms in the field of medical imaging, the backpropagation neural network (BPNN) artificial intelligence algorithm is integrated with MRI image feature analysis [13]. As early as 
2016, Chinese scholars proposed the application of BP neural network algorithm in brain tumor MRI image segmentation. His research results showed that BP neural network could effectively segment brain MRI containing fuzzy regions after strengthened training for fuzzy regions. The boundaries of the brain tumor areas were very obvious. The fuzzy areas in the lower left and right corners were mostly clustered as normal tissues, and the segmentation was relatively accurate on the whole. Artificial neural network has the ability of large-scale parallel processing and distributed information storage, good adaptive, self-organization, strong learning, association, and fault tolerance [14]. In the medical field, artificial neural network has been used in clinical diagnosis of diseases, disease screening, and auxiliary diagnosis, disease-related factors research, disease risk prediction, survival analysis, gene identification, DNA and RNA sequence analysis, protein structure analysis, etc. Artificial neural network has also been widely used in imaging, which can improve the diagnostic accuracy. In foreign countries, artificial neural network has been applied to MRI images to diagnose hip joint diseases.

To sum up, 100 patients needing total hip arthroplasty in the hospital were taken as the research objects to explore the application of MRI image feature analysis based on BPNN artificial intelligence algorithm in total hip arthroplasty under general anesthesia. They were divided into the observation group and the control group to analyze the different imaging examination results. Moreover, its effect on total hip arthroplasty was investigated in this study. The results of this study were intended to provide a reference for the application of an artificial intelligence algorithm based on BPNN in total hip arthroplasty.

\section{Methods}

2.1. Research Objects. 100 cases were selected as the research objects in this study, who were admitted to hospital during January 2018-January 2020, were diagnosed with femoral aseptic necrosis (such as femoral head necrosis), some hip fracture (such as femoral neck fractures), rheumatoid arthritis, traumatic arthritis, benign and malignant bone tumors, and ankylosing spondylitis, and required hip arthroplasty therapy. Then, they were grouped randomly into the control group and the observation group, with 50 cases in each. The general information and other conditions of patients from the two groups were compared and analyzed, and the difference was not statistically obvious $(P>0.05)$. This study was approved by the ethics committee of the hospital, and all the patients included in the study signed the informed consent forms.

The criteria for inclusion were defined to include patients who were older than 50 years old, who had the complete imaging data, whose lesions were reported, and who signed the surgical consent forms.

The criteria for exclusion were defined to include patients who were complicated with infection or other basic diseases of bone tissue, spinal cord injury, or hemodynamic instability and took part in other studies.
2.2. Examination Method. 50 patients from the control group underwent CT examinations, with the help of a CT/etype spiral CT produced by GE in the United States for scanning. The patients were placed in the supine position, and the parameters were set with the help of the CT scanner. The voltage was set as $120 \mathrm{kV}$, the tube current was set as $300 \mathrm{~mA}$, the layer thickness was set as $10 \mathrm{~mm}$, and the layer spacing was set as $10 \mathrm{~mm}$. Next, the patient's femur was scanned. Patients from the observation group were examined by MRI, and they were kept in supine position; and the femur of the patients was scanned through the MRI instrument. Using a Siemens MAGNETOM Skyra 3.0 T MRI scanner, all MRI examinations were performed prior to clinical treatment. High-resolution MRI T2 lipid-pressure sagittal scan parameters were as follows: repetition time (TR): $8500 \mathrm{~ms}$, echo time (TE): $97 \mathrm{~ms}$, field of view (FOV): $200 \mathrm{~mm} \times 200 \mathrm{~mm}$, and layer thickness: $3.5 \mathrm{~mm}$. The transverse scanning parameters of T2 lipid pressure were as follows: TR: $4400 \mathrm{~ms}$, TE: $62 \mathrm{~ms}$, FOV: $200 \mathrm{~mm} \times 200 \mathrm{~mm}$, layer thickness: $4 \mathrm{~mm}$; TR: $4300 \mathrm{~ms}$, TE: $104 \mathrm{~ms}$, FOV: $180 \mathrm{~mm} \times 180 \mathrm{~mm}$, and thickness: $4 \mathrm{~mm}$. Conventional MRI T2WI lipogenic sagittal scan parameters were as follows: TR: $5000 \mathrm{~ms}$, TE: $50 \mathrm{~ms}$, FOV: $350 \mathrm{~mm} \times 284.4 \mathrm{~mm}$, and thickness: $4 \mathrm{~mm}$. After the examinations of the patients from the two groups, surgical exploration was conducted for all patients, and the diagnostic accuracy between the groups was compared and analyzed. Its accuracy was judged by pathological biopsy results during the operation. Then, 50 patients in the observation group were divided into two groups, namely, the BP neural network group and the physician group. MRI images of patients in the former group were processed by BP algorithm, while MRI images of patients in the latter group were only diagnosed by radiologists. Finally, the sensitivity, specificity, and accuracy of the two groups of images were compared.

2.3. BP Neural Network Design. BP is a multilayer network for weight training of nonlinear differentiable functions. BPNN is a special type of BP network. BPNN consists of three layers: an input layer, a hidden layer, and an output layer. The input layer receives data and outputs data. The neurons of the previous layer connect to the neurons of the next layer to collect the information from the neurons of the previous layer and transfer the value to the next layer through "activation." The structural pattern is shown in Figure 1.

In the design of BP network, the number of grid layers, the number of neurons in each layer, the activation function, and the initial value should be considered. In this study, a three-layer BP grid with a hidden layer was adopted, and its topology was 2-3-1. The activation function of the network adopts the Sigmoid function, which is expressed as follows:

$$
f(\chi)=\log \operatorname{sig} \chi=\frac{1}{1+e^{-x}} .
$$

2.4. Training of BP Network and Simulation Test of Predictive Neural Network Model. In this study, BP neural network with forward additional momentum was adopted, and its 


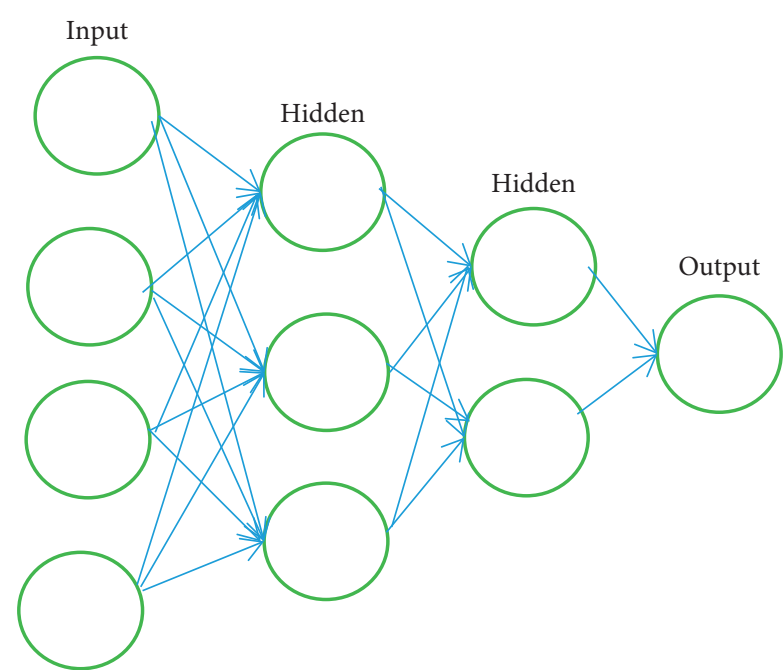

FIgURE 1: Three-layer structure pattern diagram of BPNN.

topological structure consisted of three layers: input layer, hidden layer, and output layer. There were 26 neural nodes in the input layer, including 21 neural nodes in the hidden layer with clinical parameters and above radiological features, and 6 neural nodes in the output layer using transfer function and Sigmoid linear transfer function. The steps were as follows. The input data were preprocessed. 40 groups of 50 pieces of experimental data were selected as training samples. In order to make the input data fall on the gradient with the fastest change of transfer function, preprocessing of training samples was carried out by dividing the value of each indicator by the maximum value of the indicator. The normalization equation was as follows:

$$
\chi=\frac{X i}{\max \chi} .
$$

$X i$ refers to the experimental data, and $\max (x)$ is the maximum value of each index. In order to observe the reliability of the prediction neural network model, the simulation test was carried out on the model. The remaining 10 groups of experimental data were taken as training samples, and the training samples were preprocessed. The value of each indicator was divided by the maximum value of the indicator, and the normalized equation was shown in the following:

$$
\omega=\frac{\chi}{\max (x)},
$$

where $x$ refers to the number of training samples and $\max (x)$ refers to the maximum value of each index. The trained MRI image feature prediction neural network model was applied to predict the above data and the error value was calculated. The calculation equations were as follows:

(i) Absolute error $=$ actual lesion number - predicted lesion number

(ii) Relative error $=$ absolute error/number of actual lesions $\times 100 \%$
2.5. Image Processing of BP Neural Network Algorithm. MRI images of patients' hip joint were processed using BP neural network algorithm. In order to make the input data more in line with the requirements of artificial neural network Sigmoid transfer function, linear eucalyptus tree was transformed into normalized processing, and the expression was as follows:

$$
y=\frac{(x-\text { MinValue })}{(\text { MaxValue }- \text { MinValue })},
$$

where $x$ and $y$ are the values before and after conversion, respectively, and MaxValue and MinValue are the maximum and minimum values of samples, respectively. The converted input parameters are all distributed within the range of $[0,1]$.

2.6. Staging Criteria. With the help of MRI examination, four diagnostic criteria of stage I necrosis, stage II necrosis, stage III necrosis, and stage IV necrosis were adopted for the diagnosis of patients with femoral head necrosis. Stage I necrosis: pain is in the hip, gradually forming uninterrupted hip pain that worsens as the hip is walking. Stage II necrosis: pain is usually needle-like, dull, or sore, radiating to the groin area, the inner thighs, the back of the buttocks, and the inner knee, and there will be numbness in this area. Besides, the pain will be obvious when patients are in activity, but it will be reduced after the rest. Stage III necrosis is manifested as more intense and persistent hip pain with severe joint pain while standing. Stage IV necrosis: the morphology and structure of the femoral head of the patient are significantly changed, with large areas of irregular collapse or flattening and structural variation of the trabecular bone.

2.7. Surgical Methods. The procedures of joint arthroplasty surgery were as follows: first, before the operation, the patients were under general anesthesia, and the femoral head was removed. The hip joint was successfully dislocated by flexion, adduction, and internal rotation. The femoral neck was cut off $1-1.5 \mathrm{~cm}$ above the lesser trochanter, and the round ligament and the surrounding joint capsule were cut off, so that the femoral head was removed. Second, the acetabulum was treated. Acetabular cartilage was repaired with acetabular grinding until blood oozed from subchondral bone, so as to ensure that all around the acetabulum were crushed. Acetabular prosthesis was implanted at an extroverting angle of $45^{\circ}$, and antebrachial angle of $12-15^{\circ}$ was given. Third, the femur was processed. After reaming, the medullary cavity drill from small size to large size was employed to penetrate the medullary cavity with the medullary cavity file from small size to large size. Then, reduction was tried, lining was installed, and the femoral prosthesis was implanted. Fourth, the bone cement was used for fixation and the surgical incision was closed.

2.8. Statistical Methods. Statistical software SPSS20.0 was used for statistical processing and the $X^{2}$ test was used to detect the count data. In addition, $P<0.05$ indicated a statistically significant difference. 


\section{Results}

3.1. Basic Information of Patients from the Two Groups. In this study, 100 patients needing hip arthroplasty were included in the control group $(n=50)$ and the observation group $(n=50)$, and there was no statistical difference in the basic information between the two groups $(P>0.05)$. In the control group, there were 33 male patients, accounting for $66 \%$, and 17 female patients, accounting for $34 \%$. There were 31 males (62\%) and 19 females (38\%) in the observation group. In the control group, 43 cases were more than 65 years old, accounting for $86 \%$ of the total number of patients in the control group; in the observation group, 40 cases were older than 65 years old $(80.0 \%)$. In the control group, 29 (58.0\%) had smoked, and 27 (54\%) had smoked in the observation group. There were 23 patients with a history of diabetes in the control group, accounting for $46.0 \%$, and 25 patients in the observation group, accounting for $50 \%$. In the control group, there were 17 patients with a history of heavy use of antibiotics, accounting for $34.0 \%$. The data of the two groups were not statistically significant (Table 1).

3.2. Comparison of Features of CT and MRI Images Processed by Neural Network Algorithm. CT and MRI images based on BP neural network algorithm were analyzed to judge the accuracy of these two examination methods in the diagnosis of hip diseases. The gold standard of these diseases was the result of pathological biopsy during the operation, as shown in Figure 2.

3.3. Analysis of CT and MRI Examination Results of 100 Patients. In the control group, CT examination results showed a total of 7 diseases, of which the most suggestive disease was osteoarthritis (23 cases and $46.0 \%$ ). Then, there were 17 cases of bone aseptic necrosis (necrosis of femoral head), accounting for $34.0 \%$, and 3 cases of sacrum fracture and rheumatoid arthritis, accounting for $6.0 \%$. The number of patients diagnosed with benign tumor and compulsory spondylitis was the least, 1 case each. In the observation group, MRI examination results indicated that osteoarthritis was still the most common disease (21 cases, accounting for $42.0 \%$ ), followed by aseptic necrosis of bone, sacral fracture, rheumatoid arthritis, etc. (Figures 3(a) and 3(b)).

\subsection{Comparison of Misdiagnosis Rates between CT and MRI} Examination Results of 100 Patients. The total misdiagnosis rate was $12 \%$ in the control group, including 3 cases of osteoarthritis, 2 cases of necrosis of the femoral head, and 1 case of rheumatoid arthritis, as shown in Table 2. The patients from the observation group underwent MRI examinations, and the total misdiagnosis rate was $4 \%$, including 2 patients with misdiagnosis, including osteoarthritis and aseptic necrosis of bone, as shown in Table 3.

3.5. The Comparison of the BP Neural Network Group and the Physician Group. The MRI image of hip joint after BP neural network processing is shown in Figure 4. In Table 4, MRI images processed by radiologists had higher sensitivity (\%), while images processed by BP neural network algorithm had higher specificity.

3.6. BP Network Training Results of MRI Image of Hip Joint. According to the design of neural network and the requirements of this study, the parameters were set as follows. The maximum training step epochs were 10,000; the deviation goal was 0.00001 ; the interval display during training show was 25; the maximum number of differences max-fail was 5; the number of rows and columns mem-reduc was le010; the reduction rate of characteristic function mu-dec was 0.1 ; the increase rate of characteristic function mu-inc was 10 ; the $u$ upper limit mu-max was $100,000,000,000$. Moreover, the designed neural network was trained with parameters, and the error recording curve obtained is shown in Figure 5 .

3.7. Simulation Test Results of the Prediction Neural Network Model. The data obtained after normalization of the remaining 10 groups of data were as follows: $0.89,1.01,0.45$, $0.34,1.24,0.32,1.12,0.67,0.78,0.56 ; 0.21,0.37,0.48,0.87$, $0.49,0.73,0.98,0.98,0.76,0.68 ; 0.81,0.76,0.74,0.23,0.57$, $0.76,0.35,0.85,0.26,0.83 ; 0.24,0.64,0.73,0.37,0.82,0.72$, $0.73,2.47,0.25,0.25$.

Comparison of test results with target samples is shown in Table 5.

3.8. Staging Criteria of Femoral Head Necrosis. In the process of surgical exploration, there were 17 cases of femoral head necrosis in the control group, including 7 cases of stage II femoral head necrosis $(41.2 \%), 6$ cases of stage I femoral head necrosis $(35.3 \%), 3$ cases of stage III femoral head necrosis $(11.8 \%)$, and 1 case of stage IV femoral head necrosis $(5.9 \%)$. In the observation group, there were a total of 18 cases of femoral head necrosis, of which the largest proportion was stage I ( 8 patients) $(50.0 \%)$, followed by stage II (5 cases), stage III ( 2 cases), and stage IV ( 2 cases). The accuracy of stage I diagnosis of femoral head in patients from the observation group was higher than that of the control group, and the difference was statistically substantial $(P<0.05)$ (Figures 6(a) and 6(b)).

\section{Discussion}

In clinical work, imaging methods are mainly used to diagnose bone tissue-related diseases, which mainly include X-ray plain film examination, CT examination, and MRI examination [15]. Compared with other imaging examinations, MRI examination can obtain the native 3dimensional section imaging, which also can give clear display of soft tissue and no ionizing radiation damage to human body; the MRI examination of soft tissue such as central nervous system, bladder, rectum, and uterus is better than CT, multisequence imaging, and multiple image types, providing more abundant image information for defining the nature of lesions [16]. Clinical data 
TABLE 1: Comparison of basic information of 100 patients in the control group and the observation group.

\begin{tabular}{|c|c|c|c|c|c|c|}
\hline \multicolumn{2}{|c|}{ Basic information } & $\begin{array}{l}\text { The number of cases in } \\
\text { the control group }\end{array}$ & $\begin{array}{l}\text { Proportion } \\
\quad(\%)\end{array}$ & $\begin{array}{l}\text { The number of cases in the } \\
\text { observation group }\end{array}$ & $\begin{array}{l}\text { Proportion } \\
\quad(\%)\end{array}$ & $P$ \\
\hline \multirow{2}{*}{ Gender } & Male & 33 & 66.0 & 31 & 62.0 & 0.001 \\
\hline & Female & 17 & 34.0 & 19 & 38.0 & 0.000 \\
\hline \multirow{2}{*}{ Age } & $\begin{array}{l}\text { More than } 65 \\
\text { years old }\end{array}$ & 43 & 86.0 & 40 & 80.0 & 0.002 \\
\hline & $\begin{array}{c}\text { Less than } 65 \\
\text { years old }\end{array}$ & 7 & 14.0 & 10 & 20.0 & 0.019 \\
\hline \multirow{2}{*}{ A history of smoking } & Yes & 29 & 58.0 & 27 & 54.0 & 0.011 \\
\hline & No & 21 & 42.0 & 23 & 46.0 & 0.003 \\
\hline \multirow{2}{*}{ A history of diabetes } & Yes & 23 & 46.0 & 25 & 50.0 & 0.001 \\
\hline & No & 27 & 54.0 & 25 & 50.0 & 0.001 \\
\hline \multirow{2}{*}{$\begin{array}{l}\text { A history of the heavy } \\
\text { use of antibiotics }\end{array}$} & Yes & 17 & 54.0 & 16 & 32.0 & 0.000 \\
\hline & No & 33 & 66.0 & 34 & 68.0 & 0.000 \\
\hline
\end{tabular}

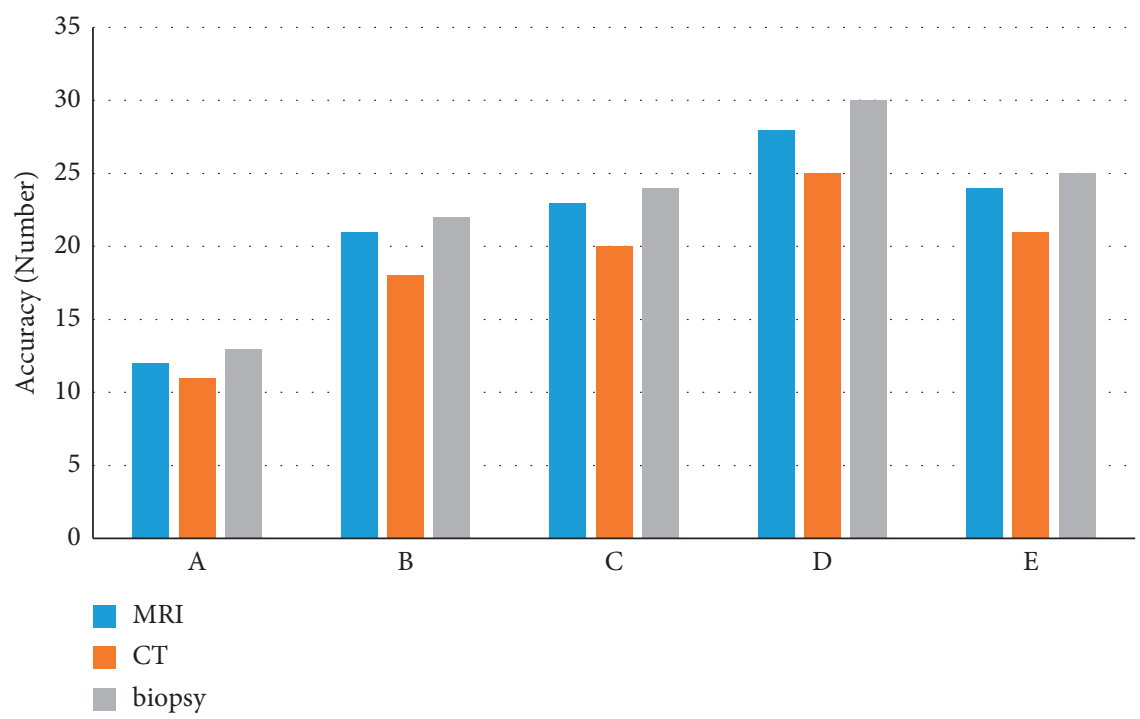

Figure 2: Comparison of diagnostic accuracy of different examination methods. Note. A: osteoarthritis; B: aseptic osteonecrosis; C: sacral fracture; D: rheumatoid arthritis; E: traumatic fracture.

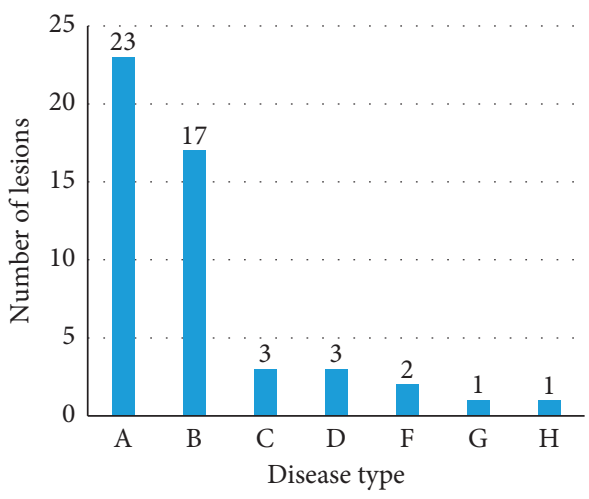

(a)

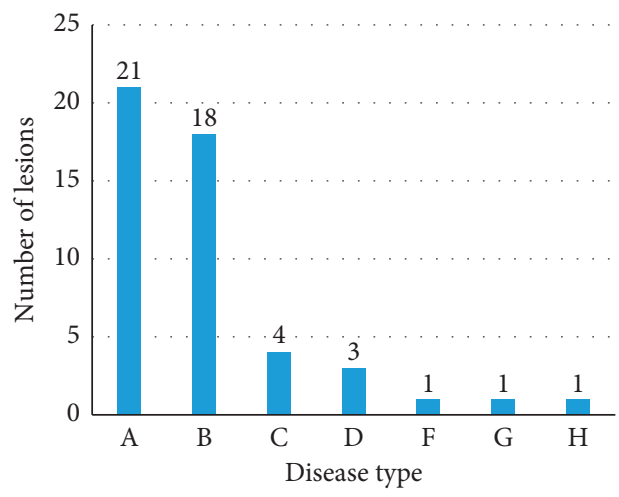

(b)

FIgURE 3: CT examination results of 50 patients in the control group (a) and 50 patients in the observation group (b). Note. A: osteoarthritis; B: aseptic necrosis of bone; C: sacral fracture; D: rheumatoid arthritis; E: traumatic fracture; F: benign or malignant tumors; G: compulsory spondylitis. 
TABLe 2: Misdiagnosis rate of CT examinations in patients from the control group.

\begin{tabular}{lccc}
\hline Control group & Actual number of cases & Number of cases diagnosed by CT & Misdiagnosis rate (\%) \\
\hline Osteoarthritis & 23 & 20 & 6 \\
Aseptic necrosis of bone & 17 & 15 & 4 \\
Sacral fracture & 3 & 3 & 0 \\
Rheumatoid arthritis & 3 & 2 & 2 \\
Traumatic fracture & 2 & 2 & 0 \\
Benign or malignant tumors & 1 & 1 & 0 \\
Compulsory spondylitis & 1 & 1 & 0 \\
Total & 50 & 44 & 12 \\
\hline
\end{tabular}

TABLE 3: Misdiagnosis rate of MRI examinations in patients from the control group.

\begin{tabular}{lccc}
\hline Control group & Actual number of cases & Number of cases diagnosed by CT & Misdiagnosis rate (\%) \\
\hline Osteoarthritis & 21 & 20 & 2 \\
Aseptic necrosis of bone & 18 & 17 & 2 \\
Sacral fracture & 4 & 4 & 0 \\
Rheumatoid arthritis & 3 & 3 & 0 \\
Traumatic fracture & 1 & 1 & 0 \\
Benign or malignant tumors & 1 & 1 & 0 \\
Compulsory spondylitis & 1 & 1 & 0 \\
Total & 50 & 48 & $4 \%$ \\
\hline
\end{tabular}

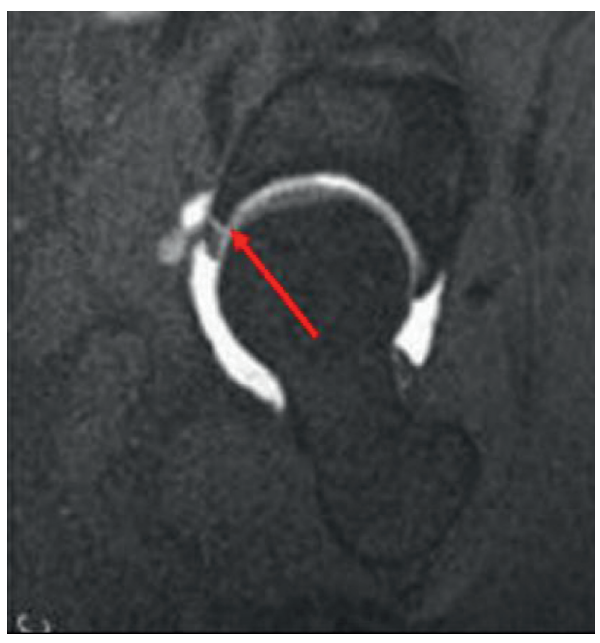

(a)

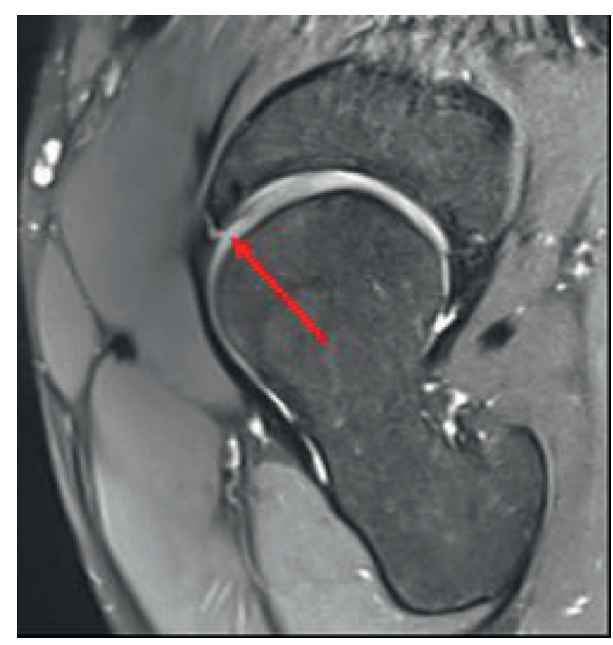

(b)

FIGURE 4: MRI image of hip joint. (a) Original MRI image and (b) image processed by BP neural network algorithm.

TABLE 4: The comparison of the BP neural network group and the physician group.

\begin{tabular}{|c|c|c|c|c|}
\hline \multirow{2}{*}{ The pathologic result } & \multicolumn{2}{|c|}{ Results of BP treatment } & \multicolumn{2}{|c|}{ Results of radiologist handled } \\
\hline & Malignant & Benign & Malignant & Benign \\
\hline Malignant & 19 & 6 & 20 & 5 \\
\hline Benign & 2 & 23 & 3 & 22 \\
\hline Sensitivity (\%) & \multicolumn{2}{|c|}{$84.0(21 / 25)$} & \multicolumn{2}{|c|}{$92.0(23 / 25)$} \\
\hline Specificity (\%) & \multicolumn{2}{|c|}{$92.0(23 / 25)$} & \multicolumn{2}{|c|}{$88.0(22 / 25)$} \\
\hline Accuracy (\%) & \multicolumn{2}{|c|}{$96.0(24 / 25)$} & \multicolumn{2}{|c|}{$92.0(23 / 25)$} \\
\hline
\end{tabular}




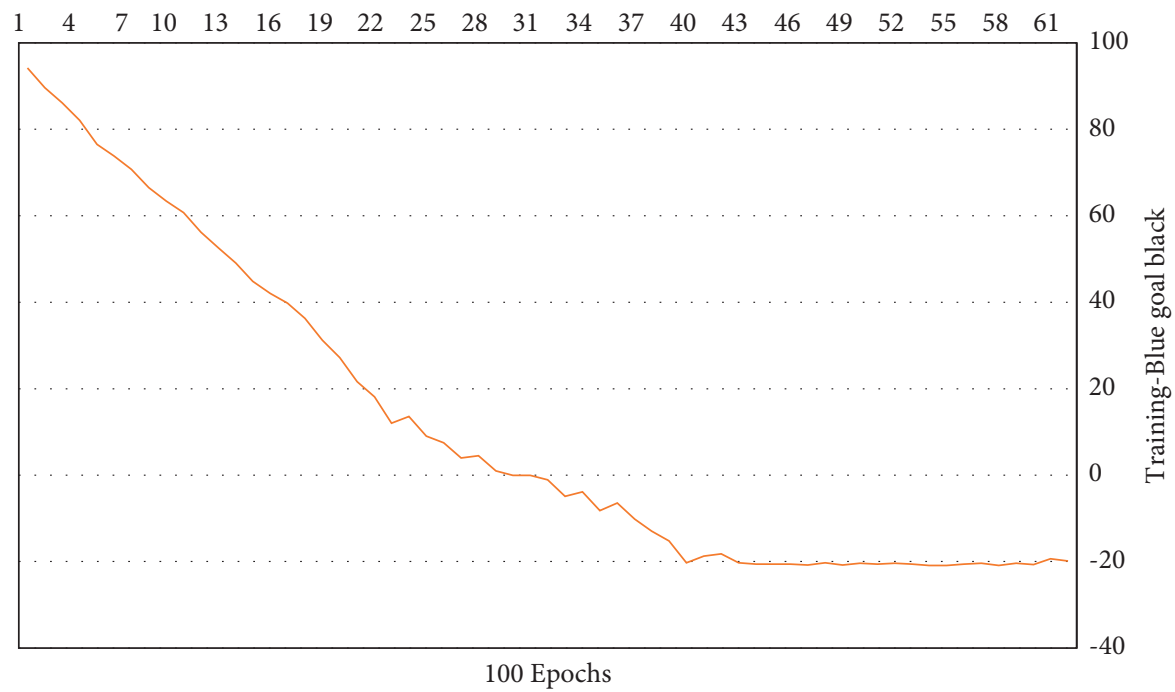

Figure 5: Training results of BP network.

TABLE 5: Comparison of MRI image prediction results with actual results.

\begin{tabular}{|c|c|c|c|c|c|}
\hline No. & Output value & Prediction of MRI image features & Actual MRI image features & Absolute error & Relative error (\%) \\
\hline 1 & 0.8638 & 78.25 & 73.36 & -4.89 & 6.37 \\
\hline 2 & 0.2789 & 39.66 & 36.36 & -3.3 & 3.25 \\
\hline 3 & 0.2566 & 39.36 & 35.29 & -4.07 & 2.36 \\
\hline 4 & 0.3672 & 63.36 & 69.35 & 5.99 & 7.34 \\
\hline 5 & 0.9735 & 29.47 & 32.53 & 3.06 & 2.56 \\
\hline 6 & 0.9857 & 29.53 & 25.46 & -4.07 & 4.36 \\
\hline 7 & 0.2978 & 58.46 & 57.36 & -1.1 & 3.56 \\
\hline 8 & 0.2256 & 37.58 & 39.45 & 1.87 & 8.56 \\
\hline 9 & 0.2789 & 43.74 & 42.54 & -1.2 & 8.46 \\
\hline 10 & 0.6583 & 27.58 & 30.52 & 2.94 & 9.45 \\
\hline Average value & 0.5186 & 55.897 & 44.22 & 0.11 & 5.73 \\
\hline Standard deviation & 0.2532 & 11.34 & 10.87 & 3.97 & 8.68 \\
\hline
\end{tabular}

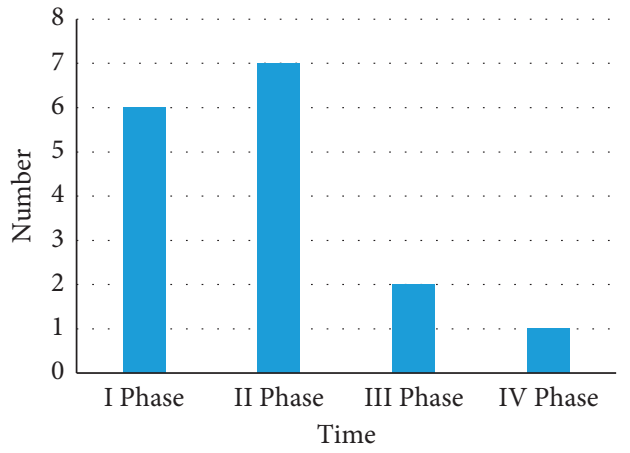

(a)

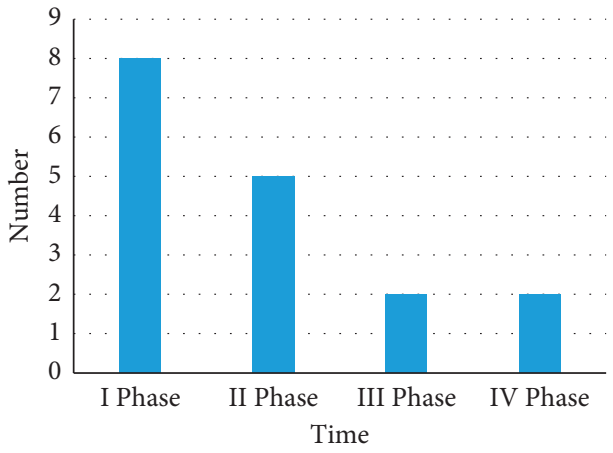

(b)

Figure 6: Staging of femoral head necrosis in the control group (a) and the observation group (b).

suggest that MRI can more specifically and clearly reflect the early necrosis of the femoral head, which has a great role in the preoperative diagnosis of hip arthroplasty [17]. The results of this study also indicated that the overall misdiagnosis rate was $12 \%$ in the control group with CT examinations and $4 \%$ in the observation group with MRI examinations, including 2 patients with misdiagnosis (osteoarthritis and aseptic necrosis of the bone), suggesting that the accuracy of MRI was higher than that of CT. 
The BPNN is adopted to analyze MRI image features, so the diagnostic rate of images can be improved. In recent years, the BPNN artificial intelligence algorithm has been integrated with MRI image feature analysis. BPNN is an artificial neural network using radial basis function as an activation function [18]. The latest research reports that $\mathrm{BPNN}$, as a kind of artificial neural network using radial basis function as an activation function, has been gradually applied in the field of medicine. BP neural network contains three layers of structure: input layer, hidden layer, and output layer [19-21]. The results of this study showed that there were absolute errors and relative errors between the predicted MRI image features and the actual MRI features. The average value of absolute error was 0.11 , and the standard deviation was 3.97, while the average value of relative error was 5.73, and the standard deviation was 8.68. After using BPNN to analyze and process the features of MRI images, the accuracy of MRI images was improved.

In clinical practice, due to osteoporosis in the elderly, trochanteric and intertrochanteric fractures of the femoral head are prone to occur, especially unstable fractures accompanied by severe osteoporosis, which eventually lead to ischemic necrosis of the femoral head [17]. Studies have shown that aseptic necrosis of the bone (necrosis of the femoral head) occurs in $23-30 \%$ of the population, and the main causes are alcohol and heavy antibiotic use. In clinical work, patients with femoral head necrosis are staged, including stages I-IV, in order to provide better surgical treatment for patients. A large number of studies have pointed out that more than $50 \%$ of the patients are in stages I and II $[22,23]$. In this study, the patients with necrosis of the femoral head in the control group and the observation group were classified. The results revealed that $41.2 \%$ of the patients from the control group were in stage II, and nearly 50\% of the patients in the observation group were in stage I, which was roughly the same as the results reported in domestic and foreign literature studies.

In this study, the BPNN artificial intelligence algorithm was applied to process the features of MRI images. The results disclosed that the misdiagnosis rate and missed diagnosis rate of MRI images processed by the BPNN algorithm were reduced, and the accuracy of MRI images was improved, thus greatly promoting the success rate of total hip arthroplasty under general anesthesia.

\section{Conclusion}

This study aimed to explore the application of MRI image feature analysis based on BPNN artificial intelligence algorithm in hip arthroplasty under general anesthesia. In this study, 100 patients who were treated in the Orthopedics Department of our hospital and needed total hip arthroplasty were selected as the research objects. Besides, they were enrolled randomly into the observation group and the control group. There was no statistical difference among the groups. It was found that MRI imaging analysis based on neural algorithm was more specific and accurate than radiologists in diagnosing MRI images. BP neural network algorithm can effectively improve the success rate of surgery in MRI diagnosis of hip diseases and reduce postoperative complications of patients. The sample size selected in this study is from a third-class A hospital, which had a small sample size. It could not accurately reflect the advantages of BP neural network algorithm in MRI. Meanwhile, this study only focuses on total hip arthroplasty. Therefore, it is necessary to explore the application of BNPP artificial intelligence algorithm in MRI image feature analysis for more surgeries. In conclusion, the results of this study can provide reference for the application value of BPNN artificial intelligence algorithm in MRI image features in surgery.

\section{Data Availability}

The data used to support the findings of this study are available from the corresponding author upon request.

\section{Conflicts of Interest}

The authors declare no conflicts of interest.

\section{References}

[1] P. Khosravi, E. Kazemi, M. Imielinski, O. Elemento, and I. Hajirasouliha, "Deep convolutional neural networks enable discrimination of heterogeneous digital pathology images," EBioMedicine, vol. 27, pp. 317-328, 2018, Epub 2017 Dec 28. PMID: 29292031; PMCID: PMC5828543.

[2] B. H. Bosker, H. B. Ettema, M. F. Boomsma, B. J. Kollen, M. Maas, and C. C. P. M. Verheyen, "High incidence of pseudotumour formation after large-diameter metal-onmetal total hip replacement," Journal of Bone and Joint Surgery British Volume, vol. 94-B, no. 6, pp. 755-761, 2012, PMID: 22628588.

[3] J. González-Zabaleta, S. Pita-Fernandez, T. Seoane-Pillado, B. López-Calviño, and J. L. Gonzalez-Zabaleta, "Comorbidity as a predictor of mortality and mobility after hip fracture," Geriatrics and Gerontology International, vol. 16, no. 5, pp. 561-569, 2016, Epub 2015 May 15. PMID: 25981487.

[4] C. Li, B. Xie, S. Chen, G. Lin, G. Yang, and L. Zhang, "The effect of local bone density on mechanical failure after internal fixation of pertrochanteric fractures," Archives of Orthopaedic and Trauma Surgery, vol. 136, no. 2, pp. 223-232, 2016, Epub 2015 Dec 1. PMID: 26626056.

[5] Y. Gilboa, T. Maeir, S. Karni et al., "Effectiveness of a telerehabilitation intervention to improve performance and reduce morbidity for people post hip fracture - study protocol for a randomized controlled trial," BMC Geriatrics, vol. 19, no. 1, p. 135, 2019 PMID: 31109289; PMCID: PMC6528189.

[6] A. Brunner, M. Büttler, U. Lehmann et al., "What is the optimal salvage procedure for cut-out after surgical fixation of trochanteric fractures with the PFNA or TFN?" Injury, vol. 47, no. 2, pp. 432-438, 2016, Epub 2015 Dec 2. PMID: 26748415.

[7] M. P. L. van der Sijp, D. van Delft, P. Krijnen, A. H. P. Niggebrugge, and I. B. Schipper, "Surgical approaches and hemiarthroplasty outcomes for femoral neck fractures: a meta-analysis," The Journal of Arthroplasty, vol. 33, no. 5, pp. 1617-1627, 2018, Epub 2017 Dec 29. Erratum in: J Arthroplasty. 2020 Feb;35(2):603-604. PMID: 29398259.

[8] J. Kuchálik, A. Magnuson, A. Lundin, and A. Gupta, "Local infiltration analgesia or femoral nerve block for postoperative pain management in patients undergoing total hip arthroplasty. a randomized, double-blind study," Scandinavian 
Journal of Pain, vol. 16, no. 1, pp. 223-230, 2017, Epub 2017 Jun 1. PMID: 28850408.

[9] G. Görmeli, M. F. Korkmaz, C. A. Görmeli, C. Adanaş, T. Karataş, and S. A. Şimşek, "Comparison of femur intertrochanteric fracture fixation with hemiarthroplasty and proximal femoral nail systems," Turkish Journal of Trauma and Emergency Surgery, vol. 21, no. 6, pp. 503-508, 2015, PMID: 27054643.

[10] E. Esen, H. Dur, M. B. Ataoğlu, T. Ayanoğlu, and S. Turanl,, "Evaluation of proximal femoral nail-antirotation and cemented, bipolar hemiarthroplasty with calcar replacement in treatment of intertrochanteric femoral fractures in terms of mortality and morbidity ratios," Joint Diseases and Related Surgery, vol. 28, no. 1, pp. 35-40, 2017, PMID: 28291437..

[11] Q. Xiao and Z. Zhou, "Perioperative pain management of total hip arthroplasty," Zhongguo Xiu Fu Chong Jian Wai Ke Za Zhi, vol. 33, no. 9, pp. 1190-1195, 2019, Chinese PMID: 31512464.

[12] A. Prestmo, G. Hagen, O. Sletvold et al., "Comprehensive geriatric care for patients with hip fractures: a prospective, randomised, controlled trial," The Lancet, vol. 385, no. 9978, pp. 1623-1633, 2015, Epub 2015 Feb 5. PMID: 25662415.

[13] A. Prestmo, I. Saltvedt, J. L. Helbostad et al., "Who benefits from orthogeriatric treatment? results from the trondheim hip-fracture trial," BMC Geriatrics, vol. 16, no. 1, p. 49, 2016 PMID: 26895846; PMCID: PMC4761133.

[14] X. Zeng, K. Zhan, L. Zhang et al., "Conversion to total hip arthroplasty after failed proximal femoral nail antirotations or dynamic hip screw fixations for stable intertrochanteric femur fractures: a retrospective study with a minimum follow-up of 3 years," BMC Musculoskeletal Disorders, vol. 18, no. 1, p. 38, 2017.

[15] J. B. Stambough, R. M. Nunley, M. C. Curry, K. Steger-May, and J. C. Clohisy, "Rapid recovery protocols for primary total hip arthroplasty can safely reduce length of stay without increasing readmissions," The Journal of Arthroplasty, vol. 30, no. 4, pp. 521-526, 2015, Epub 2015 Jan 23. PMID: 25683296; PMCID: PMC4607279.

[16] B. Nie, D. Wu, Z. Yang, and Q. Liu, "Comparison of intramedullary fixation and arthroplasty for the treatment of intertrochanteric hip fractures in the elderly," Medicine, vol. 96, no. 27, p. e7446, 2017 PMID: 28682912; PMCID: PMC5502185, Article ID e7446.

[17] A. Irajpour, M. Hashemi, and F. Taleghani, "The quality of guidelines on the end-of-life care: a systematic quality appraisal using AGREE II instrument," Supportive Care in Cancer, vol. 28, no. 4, pp. 1555-1561, 2020, Epub 2019 Dec 13. PMID: 31834517.

[18] S. Guo, R. Chen, H. Li, T. Zhang, and Y. Liu, "Identify severity bug report with distribution imbalance by cr-smote and elm," International Journal of Software Engineering and Knowledge Engineering, vol. 29, no. 02, pp. 139-175, 2019.

[19] M. Lugtenberg, K. M. van Beurden, E. P. M. Brouwers et al., “Occupational physicians' perceived barriers and suggested solutions to improve adherence to a guideline on mental health problems: analysis of a peer group training," $B M C$ Health Services Research, vol. 16, no. 1, p. 271, 2016 PMID: 27423463 ; PMCID: PMC4947285.

[20] M. C. W. Joosen, K. M. van Beurden, D. S. Rebergen et al., "Effectiveness of a tailored implementation strategy to improve adherence to a guideline on mental health problems in occupational health care," BMC Health Services Research, vol. 19, no. 1, p. 281, 2019 PMID: 31053125; PMCID: PMC6499945.
[21] E. Abarshi, J. Rietjens, L. Robijn et al., "International variations in clinical practice guidelines for palliative sedation: a systematic review," BMJ Supportive \& Palliative Care, vol. 7, no. 3, pp. bmjspcare-2016, 2017, Epub 2017 Apr 20. Erratum in: BMJ Support Palliat Care. 2018 Jun;8(2):239. PMID: 28432090 .

[22] J. Barr, G. L. Fraser, K. Puntillo et al., "Clinical practice guidelines for the management of pain, agitation, and delirium in adult patients in the intensive care unit," Critical Care Medicine, vol. 41, no. 1, pp. 263-306, 2013, PMID: 23269131.

[23] B.-W. Min, Y. Kim, H.-M. Cho et al., "Perioperative pain management in total hip arthroplasty: Korean hip society guidelines," Hip \& Pelvis, vol. 28, no. 1, pp. 15-23, 2016, Epub 2016 Mar 31. PMID: 27536639; PMCID: PMC4972874. 